\title{
High-Resolution Direct printing of Molten-Metal using Electrohydrodynamic Jet Plotting
}

\author{
Yiwei Han, Jingyan Dong* \\ Department of Industrial and Systems Engineering \\ North Carolina State University \\ Raleigh, North Carolina 27695-7906, USA
}

${ }^{*}$ Corresponding Author

Email: jdong@ncsu.edu

Tel: 919-515-7196

Fax: 919-515-5281

414-C Daniels Hall, Campus box 7906

Department of Industrial and Systems Engineering

North Carolina State University

Raleigh, North Carolina 27695-7906, USA 


\title{
High-Resolution Direct printing of Molten-Metal using Electrohydrodynamic (EHD) Jet Plotting
}

\author{
Yiwei Han and Jingyan Dong \\ Department of Industrial Engineering and Systems Engineering \\ North Carolina State University \\ Raleigh, N.C., 27695, U.S.
}

\begin{abstract}
In this paper, a high-resolution direct printing process was developed for molten metal using Elctrohydrodynamic (EHD) printing technology. The effect of the critical printing conditions, such as voltage and printing speed, on the printing process was characterized. Compared with direct extrusion using pneumatic pressure, the EHD printing can effectively reduce the dimension of the printed filament down to less than $50 \mu \mathrm{m}$ with better quality of the printed features. We successfully applied EHD printing to print high-resolution 2D patterns and some high aspect-to-ratio 3D structures, which demonstrated the potential capabilities of EHD printing process in producing fine metal structures.
\end{abstract}

Keywords: Electrohydrodynamic (EHD), electrostatic field, metal printing, 3D printing

\section{Introduction}

Additive manufacturing [1-5] has a capability of rapid prototyping and small volume production of parts for many different industries (e.g. medical device, aerospace, automotive, etc.). The resolution of tradition additive manufacturing, such as 3D printing, stereolithography, fused deposition modeling (FDM) and selective laser sintering [1-2], is approximately no better than $50 \mu \mathrm{m}$, and the improvement of the resolution from these processes is very difficult. For extrusion based printing processes, the nozzle size is the primary limitation for achieveing high printing resolution. Adopting the nozzle with too small size will result in impractically high extrusion pressure, because the extrusion pressure scales up much faster when the nozzle size is scaled down.

High-resolution metal printing is vital for many engineering areas, ranging from additive manufacturing to electronics fabrication [6-7], since high precision manufacturing of metal patterns enables the production of complex structures with reduced cost and time. A few research groups studied drop-on-demand metal printing [7-12]. The common methods for the generation of the droplet are the pneumatic-driven, piezoelectric-driven, electromagnetism-driven and laser-driven printing processes. Pneumatic driven printing uses pneumatic pressure to generate droplets [8]. Piezoelectric-driven printing 
uses a volumetric change in fluid created by the piezoelectric actuator to generate a pressure or velocity transient to print molten droplets [7,10]. The electromagnetism driven printing uses a piston that is accelerated by the magnet force to create droplets [11]. Laser-driven printing uses laser to melt thin metal layer to produce droplets [12]. Most of the current metal printing methods [7-14] are limited in resolution and the setup of the printing system is relatively complicated and expensive. New printing technology needs to be developed for high-resolution metal printing.

Electrohydrodynamic (EHD) printing [15-17] is a high-resolution printing approach, which liquid ink is subject to the electric field to form a Taylor cone and eject a droplet or jet. The size of the droplet or jet is much smaller than the size of the nozzle, which can effectively overcome the resolution limitation from the nozzle size. Currently, to the best of our knowledge, EHD printing processes were applied to polymers, metal nanoparticle inks, and biologic material [18-22], but no results has been reported on direct molten metal EHD printing.

In this work, we have successfully applied EHD printing for low melting point metal (Field's alloy) for micro-scale fabrication of metal features. High-resolution metal filaments with the smallest diameter about $50 \mu \mathrm{m}$ were successfully printed. High aspect-of-ratio 3D metal structures and metal bridges were also fabricated that have great potentials in electronics as the conductive interconnects. Compared with direct extrusion with pneumatic pressure, EHD printing significantly improves the printing resolution down to less than $50 \mu \mathrm{m}$ and provides much better quality of the printed features.

\section{Electrohydrodynamic printing system}

The EHD printing system includes four subsystems, which are a high voltage supply, a pneumatic dispensing system, a heating system and a precision three-axis motion system. The high voltage is connected to the printing nozzle, and the ground is connected to the ground electrode placed under the substrate. The pneumatic dispensing syringe is used to provide pressure for liquid flow from the printing nozzle. The syringe is heated by a heating rope with the

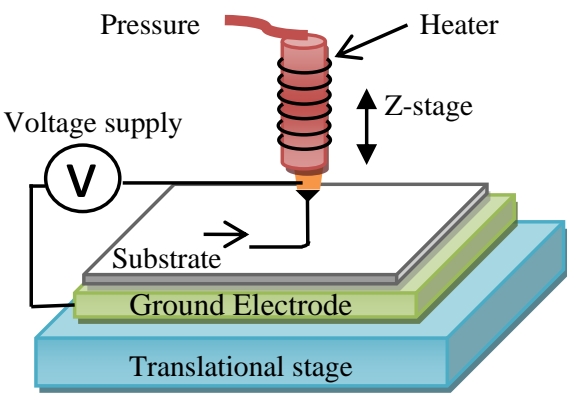

Figure 1: Schematic of EHD printing system temperature measured from a thermal couple. A PID temperature controller is used to control the syringe temperature at the desired level. The precision motion system is used to control the displacement between the printing head and the substrate in XYZ axis with an accuracy and repeatability of $100 \mathrm{~nm}$. A camera with a resolution of $0.5 \mu \mathrm{m}$ is used to monitor the printing process. The material used in this study is 
Field's Metal (32.5\% Bismuth, 51\% Indium, 16.5\% Tin), which is a eutectic alloy with a melting point of $60{ }^{\circ} \mathrm{C}$ and an electrical resistivity of $0.522 \mu \Omega \cdot \mathrm{m}$.

\section{Characterization of EHD metal printing process}

To characterize the effect of EHD printing and compare it with pneumatic extrusion-based printing, we performed printing expriments using a nozzle with an inner diameter of $160 \mu \mathrm{m}$ and an outer diameter of $250 \mu \mathrm{m}$ and keeping syringe temperature at $193{ }^{\circ} \mathrm{C}$ to achieve a good ink flowability. A standoff distance between the nozzle and the substrate is selected to be $100 \mu \mathrm{m}$ to reduce the impact of the ink stream and ink spreading on the substrate

When only applying pneumatic pressure to the syringe, for a molten metal, a large pressure is required for ink extrusion, due to the large viscous force from the molten metal. When gradually increasing the pressure, the molten metal becomes to be ejected from the nozzle and forms a droplet at the nozzle tip because of the large surface tension force and viscous force of the molten metal. The size of the droplet continues to grow when the pressure is gradually increased, and detaches from nozzle to form a bulk drop. As can be observed from Figure 2 (a), the printed droplets are at the scale of milimeters, which is much large than the nozzle dimension. After further increasing the pressure to a threshold level, which is $10 \mathrm{psi}$ in our experiment, continuous jet will form and be printed from the nozzle. The jetting speed of the molten Field's alloy is very fast. The stage speed needs to match the jet speed to achieve the uniform line and avoid the distortion (breaking line, wave line, etc.). The extruded metal lines have diameters of a few millimeters, as shown in Figure 2(b, c). Overall, the direct pneumatic extrusion cannot provide high printing resolution, and the feature unifromaity is not satisfacory.


Figure 2:(a) Pneumatically extruded droplet under pressure of 5psi. (b) Printed metal lines at a pressure of 10psi and speed of $20 \mathrm{~mm} / \mathrm{s}$. (c) Printed metal line with a pressure of $10 \mathrm{psi}$ and speed of $40 \mathrm{~mm} / \mathrm{s}$. 


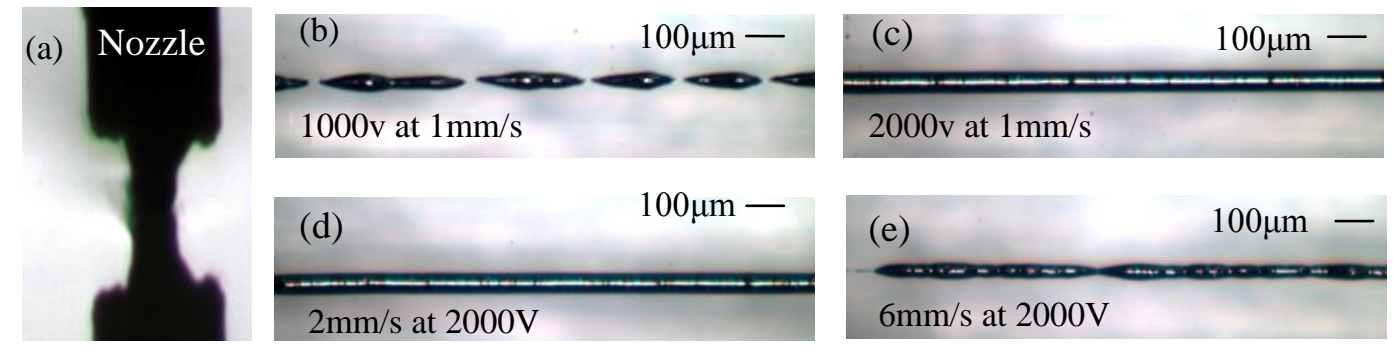

Figure 3: (a) Cone shape during the EHD printing process. (b) and (c) Printed metal line at printing voltage of $1000 \mathrm{v}$ and $2000 \mathrm{v}$ with a fixed printing speed of $1 \mathrm{~mm} / \mathrm{s}$. (d) and (e) Printed metal line at printing speed of $2 \mathrm{~mm} / \mathrm{s}$ and $6 \mathrm{~mm} / \mathrm{s}$ with a fixed printing voltage of $2000 \mathrm{v}$

To achieve better printing resolution, EHD printing is applied for the fabrication of the molten Field's Metal. Applying a voltage to the ink produces an electrostatic force, which pulls the ink out from nozzle to form a cone shape (i.e. Taylor Cone) and ejects a filament from the Taylor cone at the high enough voltage, as shown in Figure 3(a). When the applied voltage is large enough, even without the pneumatic pressure, the electrostatic force can overcome the surface tension and viscous force of the molten metal and eject the ink onto the substrate. The experiments were performed to characterize the EHD printing process and find the working voltage for the stable EHD printing. When the applied voltage (i.e. $1000 \mathrm{~V}$ in this setup) is not large enough, a discontinuous metal line is printed on the substrate (Figure $3 b$ ), which comes from small ink flow rate due to inadequate electrostatic force. After further increasing the voltage to $2000 \mathrm{v}$, a fine straight line with a small diameter of less than $50 \mu \mathrm{m}$ is achieved (Figure $3 \mathrm{c}$ ). Printing speed is the other critical factor that will affect the printing results. When keeping a constant voltage (2000v in this experiment), low printing speed will result in material accumulation at the nozzle tip and large line width of printed feature, as shown in Figure 3(d). Too high printing speed will cause line necking and discontinuity of the printed features (Figure 3e)).

\section{$4 \quad$ High resolution EHD printing of metal structures}

Using EHD printing process, we successfully fabricated high resolution 2D and 3D metal features. For 3D printing, the temperature of the molten metal needs to be selected carefully, so that the printed metal ink solidifies quickly without re-melting the previously printed structures. Figure 4(a) shows a directly printed 2D metal pattern, which has very uniform line width, about $80-90 \mu \mathrm{m}$. The overall resistance of this $2 \mathrm{D}$ pattern is about $20 \mathrm{ohms}$, which gives a resistivity of $0.6 \mu \Omega \mathrm{m}$ and has good potentials to be used in the direct fabrication of interconnects in electronics. Figure 4 (b) shows a twolayer scaffold structure by EHD metal printing. By printing along the vertical direction, high aspect of ratio pillar was directed printed onto the substrate. Figure 4(c) shows a printed vertical metal pillar with the diameter about $90 \mathrm{um}$, and the height about $10 \mathrm{~mm}$, which give an aspect-to-ratio as high as 100 . With 
proper overlap of filaments and repeating the printing sequences layer by layer, 3D structures can be printed directly with good resolution. Figure 4(d) demonstrate the printed thin wall structure with the wall thickness of $100 \mu \mathrm{m}$ and wall height of about $3 \mathrm{~mm}$. Moreover, direct EHD metal printing can create bridging metal structures, as shown in Figure 5(e), which has potential applications in wire bonding and high quality interconnects in chipboard manufacturing and electroncis manufacturing. Compared with the the best feature resolution (X/Y resolution
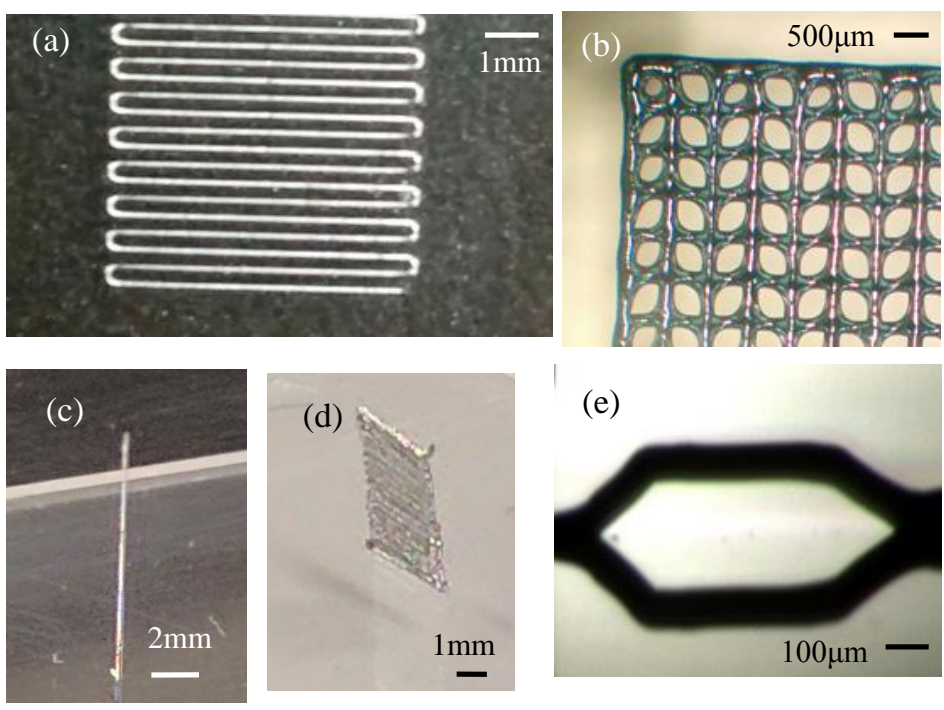

(e)

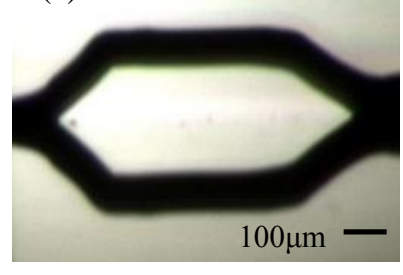

Figure 4: (a) Metal wire (b) Microscope image of two layer scaffold structure (c) Vertical metal wire (d) Thin wall (e) Microscope image of two adjacent layer of thin wall (f) metal bridge structure of 0.012- 0.016 inch or 305-406 $\mu \mathrm{m}$ )

from the start-of-art 3D metal printing machines, such as Stratasys Direct, Inc., the EHD metal printing offer much better resolution capabilities.

\section{Conclusions}

In this study, a direct molten metal EHD printing process was developed. The effect of the critical printing conditions, such as electrostatic field and printing speed, on the printing process was characterized, and the representative results were demonstrated. In EHD printing, the resulting electrostatic force can continuously print the molten metal even without using any pneumatic pressure. Compared with direct extrusion using pneumatic pressure, the EHD printing can effectively improve the printing resolution to better than $50 \mu \mathrm{m}$ and achieve better quality of the printed features. We successfully applied EHD printing to print high-resolution 2D patterns and high aspect-to-ratio 3D structures, which demonstrated its potential capabilities in producing fine metal structures.

\section{Acknowledgement}

This work was supported in part by the National Science Foundation under Grant CMMI-1333775, and CBET-1344618. 


\section{References}

[1] Gibson, I., Rosen, D., \& Stucker, B. (2009) Additive manufacturing technologies: rapid prototyping to direct digital manufacturing. Springer.

[2] Kruth, J. P., Leu, M. C., \& Nakagawa, T. (1998). Progress in additive manufacturing and rapid prototyping. CIRP Annals-Manufacturing Technology, 47(2), 525-540.

[3] Melchels, F. P., Domingos, M. A., Klein, T. J., Malda, J., Bartolo, P. J., \& Hutmacher, D. W. (2012). Additive manufacturing of tissues and organs. Progress in Polymer Science,37(8), 10791104.Koestler, A., 1989. The Ghost in the Machine. London: Arkana Books.

[4] Beaman, J., Harris L. Marcus, David L. Bourell, Joel W. Barlow, Richard H. Crawford, Kevin P. McAlea. "Solid freeform fabrication: a new direction in manufacturing." Kluwer Academic Publishers, 1997.

[5] Hutmacher, D. W., Sittinger, M., \& Risbud, M. V. (2004). Scaffold-based tissue engineering: rationale for computer-aided design and solid free-form fabrication systems. TRENDS in Biotechnology, 22(7), 354-362.

[6] Park, B. K., Kim, D., Jeong, S., Moon, J., \& Kim, J. S. (2007). Direct writing of copper conductive patterns by ink-jet printing. Thin Solid Films, 515(19), 7706-7711.

[7] Liu, Q., \& Orme, M. (2001). High precision solder droplet printing technology and the state-of-theart. Journal of materials processing technology, 115(3), 271-283.

[8] Wang, C., Liao, D., Liu, S., Li, X., \& Zhang, H. (2016, August). Effect of droplet spacing \& deposition height on $\mathrm{Sn}$ conductive lines by pneumatic diaphragm drop-on-demand technology. In Electronic Packaging Technology (ICEPT), 2016 17th International Conference on (pp. 15121516). IEEE.

[9] Wang, C. H., Tsai, H. L., Wu, Y. C., \& Hwang, W. S. (2016). Investigation of molten metal droplet deposition and solidification for 3D printing techniques. Journal of Micromechanics and Microengineering, 26(9), 095012.

[10] Orme, M., Liu, Q., \& Smith, R. (2000). Molten aluminum micro-droplet formation and deposition for advanced manufacturing applications. Aluminum Transactions, 3(1), 95-103.

[11] Kessling, O. S., Werner, K., Irlinger, F., \& Lüth, T. C. (2010, December). Indium solder printing for low temperature applications and modeling of a droplet generator. In Robotics and Biomimetics (ROBIO), 2010 IEEE International Conference on (pp. 833-838). IEEE. 
[12] Zenou, M., Sa'ar, A., \& Kotler, Z. (2015). Laser jetting of femto-liter metal droplets for high resolution 3D printed structures. Scientific reports, 5 .

[13] Wang, L., \& Liu, J. (2014). Liquid phase 3D printing for quickly manufacturing conductive metal objects with low melting point alloy ink. Science China Technological Sciences, 57(9), 1721-1728.

[14] Hsieh, P. C., Tsai, C. H., Liu, B. H., Wei, W. C. J., Wang, A. B., \& Luo, R. C. (2016, March). 3D printing of low melting temperature alloys by fused deposition modeling. In 2016 IEEE International Conference on Industrial Technology (ICIT) (pp. 1138-1142). IEEE.

[15] Park Jang-Ung, Hardy Matt, Kang Seong Jun, Barton Kira, Adair Kurt, Mukhopadhyay Deep Kishore, et al. High-resolution electrohydrodynamic jet printing. Nat Mater 2007;6(10):782-9.

[16] Mishra, S., Barton, K. L., Alleyne, A. G., Ferreira, P. M., \& Rogers, J. A. (2010). High-speed and drop-on-demand printing with a pulsed electrohydrodynamic jet. Journal of Micromechanics and Microengineering, 20(9), 095026.

[17] Poellmann, M. J., Barton, K. L., Mishra, S., \& Johnson, A. J. W. (2011). Patterned hydrogel substrates for cell culture with electrohydrodynamic jet printing. Macromolecular bioscience, 11(9), 1164-1168.

[18] Wei, C., Qin, H., Ramírez-Iglesias, N. A., Chiu, C. P., Lee, Y. S., \& Dong, J. (2014). High-resolution ac-pulse modulated electrohydrodynamic jet printing on highly insulating substrates. Journal of Micromechanics and Microengineering, 24(4), 045010.

[19] Wei, C., \& Dong, J. (2014). Hybrid hierarchical fabrication of three-dimensional scaffolds. Journal of Manufacturing Processes, 16(2), 257-263.

[20] Wei, C., \& Dong, J. (2013). Direct fabrication of high-resolution three-dimensional polymeric scaffolds using electrohydrodynamic hot jet plotting. Journal of Micromechanics and Microengineering, 23(2), 025017.

[21] Han, Y., Wei, C., \& Dong, J. (2015). Droplet formation and settlement of phase-change ink in high resolution electrohydrodynamic (EHD) 3D printing. Journal of Manufacturing Processes.

[22] Han, Y., Wei, C., \& Dong, J. (2014). Super-resolution electrohydrodynamic (EHD) 3D printing of micro-structures using phase-change inks. Manufacturing Letters, 2(4), 96-99. 